\title{
Guest editorial: multiprocessor scheduling
}

\author{
Alan Burns • Laurent George
}

Published online: 24 January 2013

(C) Springer Science+Business Media New York 2013

Real-time multiprocessor systems have an increasing importance in current technology and are the source of innovations to build new applications that require high levels of computations (in for example, industrial processes, transportation, telecommunications, multimedia and medical equipment). In the context of real-time systems, it is not sufficient to prove the correctness of applications in term of results but also that those results are delivered in time. The following papers are extended, peer-reviewed, journal-versions of papers focusing on multiprocessor systems that have been selected by the conference program committee of the 19th Real-Time and Network Systems Conference (RTNS'2011) for their high quality. RTNS'2011 was held in Nantes, France in September 2011.

The annual RTNS conference is mainly focused on the following topics: realtime system design and analysis; infrastructure and hardware for real-time systems; and real-time applications in the automotive, avionics, telecommunications, process control and the multimedia industries.

Each paper was extended to add significant extra material and the resulting papers were re-reviewed and subject to the usual editorial process. The result is a special issue that we hope will be of interest to the real-time community.

In recent years, multiprocessor architectures have become a firm favorite with the real-time community, and multicore processors are found in products ranging from small portable cell phones to large computer servers. New scheduling approaches

\footnotetext{
A. Burns

University of York, York, England, UK

e-mail: alan.burns@york.ac.uk

L. George ( $\varangle)$

University of Paris-Est, Marne La Vallée, France

e-mail: lgeorge@ieee.org
} 
must be proposed to fully exploit the capabilities of multiprocessor platforms and to go beyond classical identical processor platforms. New design techniques, scheduling approaches, real-time analysis methods and real-time operating systems have to be developed. In this issue, we first focus on real-time scheduling on Graphics Processing Units, a specialized electronic circuit designed to rapidly manipulate and alter memory to accelerate the building of images. We then explore the use of formal verification techniques to decide on the schedulability of sporadic tasks on identical multiprocessor platforms. With the evolution of processor platforms, unrelated processors are in the mainstream to specialize computations for better performances. The third paper proposes a polynomial approximation scheme for sporadic tasks upon unrelated multiprocessors (which is known to be a NP-hard problem in the strong sense). The last paper explores the use of parallel scheduling to take advantage of multiprocessor platforms. Those four papers are now introduced in more details.

The first paper, "An Optimal $k$-Exclusion Real-Time Locking Protocol motivated by Multi-GPU Systems", is by Glenn A. Elliot and James H. Anderson. It explores the use of Graphics Processing Units (GPUs). They propose a $k$-exclusion locking protocol to arbitrate access to shared resources (at most $\mathrm{k}$ task can lock a resource), in the context of pools of GPU resources where each GPU is executing program code non-preemptively. They explore global job level static-priority scheduling (G-EDF is one example). They develop the O-KGLP, a $k$-Exclusion Global Locking Protocol, that is asymptotically optimal w.r.t. the number of resource requests that may block one another. They compare the performances of O-KGLP to the $k$-FMLP ones. They show that O-KGLP is able to schedule more task sets than $k$-FMLP.

The second paper is "Multiprocessor Schedulability of Arbitrary-Deadline Sporadic Tasks: Complexity and Antichain Algorithm" by Gilles Geeraerts, Joël Goossens and Markus Henrik Lindstrom. It explores the use of a finite automaton state reachability problem to decide on the schedulability of arbitrary-deadline sporadic task sets on identical multiprocessor platforms. They characterize the complexity of this problem by proving that it is PSPACE-complete and then use the antichain formal verification technique to the Baker and Cirenei automaton, to detect the reachability of failure states in the automaton. A failure state is a state where a task misses its deadline. They show through implementation and experimental analysis that their algorithm outperforms Cirenei's original brute-force algorithm.

The third paper is "Partitioned EDF scheduling on a few types of unrelated multiprocessors" by Andreas Wiese, Vincenzo Bonifaci and Sanjoy Baruah. It proposes a polynomial-time approximation scheme (PTAS) for the partitioned EDF scheduling of implicit-deadline sporadic task upon limited unrelated multiprocessors, where the number of distinct processors is relatively small. Even if the general problem of partitioning implicit-deadline sporadic task systems on unrelated platforms is not in PTAS, they show that for high number of cores when all cores are only of a few distinct core types, the partitioning problem is in PTAS.

The last paper is "Scheduling of Hard Real-Time Multi-Phase Multi-Thread (MPMT) Periodic Tasks", by Pierre Courbin, Irina Lupu and Joël Goossens. It introduces a new parallel task model which allows recurrent (periodic) tasks to be 
composed of several phases on a set of identical multiprocessors. Each phase is composed of several parallel threads, each thread being executed on a single processor. They show how to transform a classical fork-join task model to a MPMT task model. Then, they propose two exact schedulability tests for hierarchical and global thread scheduling.

In summary, the contents of this special issue reflects the current research trends in real-time computing, which include scheduling for GPUs, formal verification approaches for real-time systems, polynomial time approximations schemes for unrelated processors and parallel scheduling for multi-phase multi-thread tasks. We hope you find this special issue interesting. 